Ophthalmologe 2022 119 (Suppl 2):S152

https://doi.org/10.1007/s00347-022-01583-w

Online publiziert: 31. Januar 2022

(C) The Author(s), under exclusive licence to Springer Medizin Verlag GmbH, ein Teil von Springer Nature 2022

\section{Erratum zu: Stellungnahme der Rechtskommission des BVA und der DOG zur augenärztlichen Beurteilung im Schwerbehindertenrecht und bei Blindheit}

Berufsverband der Augenärzte Deutschlands e. V. (BVA) ${ }^{1}$ Deutsche Ophthalmologische Gesellschaft (DOG) ${ }^{2}$

'Berufsverband der Augenärzte Deutschlands e. V., Düsseldorf, Deutschland

${ }^{2}$ Deutsche Ophthalmologische Gesellschaft (DOG), München, Deutschland
Erratum zu:

Ophthalmologe 2021

https://doi.org/10.1007/s00347-021-

01550-x

Die in dem ursprünglichen Artikel genannten Autoren waren falsch. Die korrekte Autorenzeile des Beitrags lautet Berufsverband der Augenärzte Deutschlands e.V. (BVA) ${ }^{1}$ - Deutsche Ophthalmologische Gesellschaft (DOG) ${ }^{2}$

1 Berufsverband der Augenärzte Deutschlands e. V., Düsseldorf, Deutschland

2 Deutsche Ophthalmologische Gesellschaft, München, Deutschland

Die Online-Version sowie das PDF des Beitrags wurden nachträglich korrigiert. Bitte beachten Sie die korrigierte Autorenzeile.

Die Redaktion

\section{Korrespondenzadresse}

Deutsche Ophthalmologische Gesellschaft (DOG)

Deutsche Ophthalmologische Gesellschaft (DOG)

Platenstr. 1, 80336 München, Deutschland geschaeftsstelle@dog.org
Die Online-Version des Originalartikels ist unter https://doi.org/10.1007/s00347-021-01550-x zu finden. 